Published in final edited form as:

J Cross Cult Psychol. 2009 November 1; 40(6): 909-916. doi:10.1177/0022022109349172.

\title{
Qualitative and Mixed Methods Research in Cross-cultural Psychology: Introduction to the Special Issue
}

\author{
Alison Karasz, PhD and \\ Department of Family Medicine Albert Einstein College of Medicine \\ Theodore M. Singelis, PhD \\ Department of Psychology California State University, Chico
}

Throughout most of its history it would have been unlikely that the Journal of Cross Cultural Psychology would have shown interest in a special issue devoted to qualitative and methods. Indeed we were surprised by the tremendous support and enthusiasm shown by JCCP editors when we first proposed the idea for this project in the summer of 2006. But today it is evident that qualitative and mixed methods research is enjoying a renaissance in psychology, as in the other social and health sciences. Signs of a resurgence are everywhere, ranging from a report from the United States National Institutes of Health encouraging the submission of qualitative applications (Office of Behavioral and Social Sciences Research, 1999), to the emergence of new journals emphasizing qualitative and mixed methods, to the establishment of the American Psychological Association's new division of qualitative research.

Of course, the interest is hardly new. Wilhelm Wundt, the founder of experimental psychology, proposed qualitative methods for the study of psychology in its cultural context and wrote a vast tome, Voelkerpsychologie, on the subject (Wundt, 2008). Even as the twentieth century progressed, researchers in experimental, personality, and clinical psychology continued to use qualitative and mixed methods, though they may not have articulated them as such (Ashworth, 2003). Yet over the past fifty years, qualitative research in psychology has been largely marginalized. Qualitative researchers have been barred from large research departments and have found it difficult to publish their work in major journals. Mixed methods research has fared even less well, although it is now beginning to re-establish itself in the applied sub-disciplines such as educational and industrial psychology.

The origin of psychology's marginalization of qualitative inquiry has been the focus of several historical accounts (Ashworth, 2003; Tesch, 2009) and doubtless reflects larger forces shaping the modern history of the social sciences. However, a key proximal cause of the isolation of qualitative research was the development, at mid-century, of the "incompatibility hypothesis." This hypothesis asserts that qualitative and quantitative research paradigms are inherently incompatible — differing not only in their strategies of inquiry, but also by their research goals, and, most importantly, by the epistemologies in which they are anchored. According to the hypothesis quantitative research is inseparable from a "positivist" epistemology, while qualitative research is inherently inseparable from a "constructivist," or "naturalist" epistemology (Tesch, 2009; Maxcy, 2003). In this view, quantitative, empiricist research attempts to gauge reality as it really is; constructivist research seeks to describe the "realities" constructed by subjects.

The incompatibility hypothesis escalated into an acrimonious conflict-the "paradigm wars"--that raged furiously throughout the nineteen seventies and eighties. Warriors on both sides declared not only the impossibility of dialogue, but also the irreducible superiority of 
their own position. By the late eighties, however, a softening in relations between the two camps began to emerge. Over the past fifteen years, a new generation of mixed methods researchers is declaring the paradigm wars over, having been superseded by the "third methodological way"(Maxcy, 2003; Teddlie \& Tashakkori, 1998; Johnson \& Onwuegbuzie, 2009). This third way asserts the "compatibility hypothesis," claiming "the objectives, scope, and nature of inquiry [in qualitative and quantitative research] are consistent across methods and across paradigms."(Johnson et al., 2009) The third way is grounded in the philosophy of pragmatism (Teddlie et al., 1998) — and a practical epistemology in which the idea of "what is true" is closely linked to the reality of "what works." In the context of research methodology, a pragmatic perspective proposes that research methods and designs must be judged on the basis of what they can accomplish. The special issue aims to demonstrate this position - the "third way"-for cross-cultural psychology.

Our criteria in choosing papers for this special issue were grounded in a pragmatic perspective. First, we sought reports of studies that investigated research questions of interest to cross-cultural psychologists. Second, we sought studies in which design and method were appropriate for the research question being asked. We were especially interested in comparative studies since comparative research is one of the hallmarks of cross-cultural psychology. As you look over the papers presented in this issue you will find that they do not all fit neatly into traditional categories. Some of them privilege individual perspectives and experiences (Lim; Kurman \& Roer-Strier); others do not (e.g. Keller \& Otto). One involves the quantification of qualitative data and hypothesis testing (Karasz). Several studies rely on interview data (Karasz; Penny, Newton \& Larkin) and several employ observation (Greenfield, Maynard, and Marti; Keller \& Otto). Two papers use narrative analysis, focusing less on what is said by participants than on how they say it (Weisman; Nalkur).

We were especially motivated to include high quality mixed methods studies in the issue. Several uses of mixed methods are illustrated, including the quantitative first-qualitative second design (Kurman \& Roer-Strier) and the use of alternating methods in a long-term research program (Keller \& Otto). If we had to characterize the epistemological position underlying most of the studies, we would probably use the term "soft empiricism". By this we mean that while the subjective worlds of research participants are a central focus, these "inner worlds" are investigated in order to shed light on specific problems in the "real" world. For example, Penny, Newton and Larkin identify a public health problem: the failure of the mental health system to provide effective care to ethnic minority groups. The authors analyze interview data with the goal of understanding how culturally shaped models of illness and care may act as a barrier to treatment engagement. This pragmatic, empiricist focus is common in many good qualitative studies.

We will now turn to a more general discussion of qualitative and mixed methods research. Anticipating that some readers may lack experience with these methods, we present below brief descriptions of some of their typical features. We also point out some of the common fictions associated with these approaches.

\section{What is qualitative and mixed methods research?}

\section{Qualitative research is descriptive-partly fact}

It is true that qualitative designs, which often involve prolonged contact between researcher and the field, are uniquely suited the generation of in depth and detailed descriptions. When little is known of a phenomenon, pure description may be of high intellectual and practical value. An example of the appropriate use of a descriptive approach may be found in Lim's study reported in this issue. Lim investigates a little known phenomenon: the experience of 
transnationalism in a group of Somali refugees. Previous research on transnationalism has been limited to documenting the flow of resources across borders. Lim's focus on personal experience and affective ties helps to explain a poorly understood phenomenon: why impoverished refugees support families and communities in their home country. Unfortunately, many descriptive qualitative studies published in the literature do little to address gaps in knowledge or generate new understanding. Research that remains "stuck" at the descriptive level often generates weak analyses lacking in novelty which have little power to shape our understanding of the world.

\section{Qualitative research is concerned with subjectivity- fact}

As we have already noted, qualitative research is frequently concerned with subjective meanings. Because subjective meaning is best understood through dialogue with the subject, qualitative research methods are arguably superior to questionnaire methods for understanding subjectivity. Subjectivity is an important focus in qualitative research for several reasons. First, it is of arguable moral and political value to explore the perspectives of marginalized groups. For this reason participatory action research, which seeks to draw on the perspectives and meet the needs of communities, tends to draw on qualitative and mixed methods in participatory research designs. Second, as noted, the perspective of research participants can often provide interesting insights into why people do what they do, a subject that should be of considerable interest to psychologists.

However, though self-report data can provide revealing insights into the mysteries of behavior, good qualitative research never confuse subjective report with actual fact. Good qualitative research is appropriately critical of interview and self-report data, recognizing that participants rarely know exactly why they think, feel or behave as they do, and that both inadvertent and intentional lying are the rule rather than the exception. A variety of techniques can be used to validate participant reports. For example, the use of "purposive" sampling strategies in which participants with different perspectives are systematically sampled can help in this regard. Another promising strategy is the use of multiple data collection strategies within the same study. Supplementing interview data with observational, archival, or other data can be helpful in revealing the biases in self-report.

Another strategy for "problematizing" subjective accounts lies in the techniques of narrative analysis. Two papers presented in the special issue (Weisman and Nalkur) use narrative analysis techniques to interpret stories of research subjects. Weisman examines immigration stories among immigrants from the Soviet Union to Israel, comparing two groups of immigrants: those who define their immigration experience as successful and those who do not. Her analysis shows how similar experiences across the two groups-especially humiliations and obstacles - are interpreted in a completely different light depending on the story teller's sense of personal success or failure. Weisman's analysis problematizes participants' accounts, demonstrating how narratives constitute an often unconscious selfportrait of the speaker. Similarly, Nalkur compares stories of achievement among three groups of Tanzanian children: school children, street children, and former street children. She shows how the children's differing social worlds shape the form and contents of their stories, reflecting strategies of survival and achievement adapted to the constraints unique to each context.

\section{Qualitative research is subjective-fiction}

Although it is true that qualitative researchers may be more likely to articulate sources of bias-and to worry about them--than quantitative researchers, most empirically oriented qualitative and mixed methods researchers do not agree with the common view that qualitative research is inherently more subjective than quantitative research. In quantitative 
research the choice of the construct, method, sample, the design of instruments, the choice of research setting all reflect the biases of the researcher (Johnson et al.,). These researcher biases shape the study before the data are collected. In qualitative studies, by contrast, bias often enters the study at a later point-- the data analysis phase. To minimize this problem, many qualitative researchers utilize technical strategies (Johnson et al., 2009). These include reflexivity exercises, in which members of an analysis team document their biases before beginning the analyses (Barry, Britten, Bradley, \& Stevenson, 1999), member checking, in which analysis results are presented to research participants for review (Lincoln \& Guba, 1985), and the systematic search for cases that do not fit the emerging model or theory (Strauss \& Corbin, 1998).

\section{Causality is not a concern in qualitative research-fiction}

Although researchers in some purely constructivist traditions are largely concerned with description, many other qualitative researchers unapologetically seek to generate explanations of reality (Johnson et al., 2009). For example grounded theory, one of the best known and carefully articulated qualitative traditions, emphasizes the development of explanatory theory. In applied disciplines such as educational psychology, qualitative and mixed methods researchers routinely utilize qualitative methods to address questions of causality. A typical example is the use of comparative case study methods to examine the effects of educational interventions. In typical studies of this type, quantitative data (changes in student test scores) provide the metric for the effectiveness of the interventions, while qualitative data on implementation and process explain how and why interventions succeed or fail.

It is true that qualitative researchers employ different strategies from those of quantitative researchers to arrive at explanations of phenomena. The " $w h y$ " that is addressed through statistical methods of analysis concerns the "why" of association. The goal of this research is to establish regularities in data, which can lead to the inference of cause. (Mohr, 1982; Maxwell, 2004). The "why" of qualitative research, by contrast, may be characterized as the why of process. Because qualitative researchers may be present in the research field over time, they have opportunity to experience the events and mechanisms that create change and development (Mohr, 1982) (Maxwell \& Loomis, 2003; Maxwell, 2004). Contrary to the common view that qualitative research cannot explain phenomena, some philosophers in the history of science have proposed that qualitative research is uniquely suited to the identification of causal processes. Qualitative research identifies processes of mechanical or physical causality as opposed to the descriptive or correlational causes identified in quantitative research (Mohr, 1982; Maxwell, 2004).

\section{Why does cross-cultural psychology "need" qualitative and mixed methods?}

We propose that the time has come for cross-cultural psychology, as a discipline, to embrace mixed methods research. Indeed, we argue that cross cultural psychology needs mixed methods research, and that a creative embrace of these new approaches will address some of the specific problems currently facing our discipline. A few of these problems are discussed below.

\section{The problem of culture}

In recent years cross-cultural psychologists and others (Chasiotis, Breugelmans \& van de Vijver, 2009) have increasingly called for greater coherence to the approach to culture. In conventional cross-cultural psychology research designs, culture is often conceptualized as a category - a grouping variable-for making comparisons. The contents, processes, and 
structures that constitute culture are not specified in these designs. As a result, the findings of psychological differences in such studies are peculiarly uninformative (Ratner \& Hui, 2003; Chasiotis et al., 2009). This lack of attention to concrete contents of culture is undoubtedly due, in part, to the historical interest among cross cultural psychologists in establishing abstract, universal principals governing the relationship between culture and psyche. Although there is nothing wrong with universal laws, it has been argued that we still know too little of either culture or psyche to posit universal laws that are actually informative. As a consequence, scholars have called for reducing the level of abstraction at which culture is currently conceptualized (Adamopoulos \& Lonner, 2001). This means more attention to concrete, specific, local cultural processes that shape psychological outcomes in which cultural psychologists are interested.

Qualitative research is particularly suited to this task. Observational and interview methods can generate the kinds of descriptive data that render culture more concrete and local. Fieldwork can help to identify local social and cultural processes as they unfold over time. Such approaches help to identify the mechanisms (Maxwell, 2004) through which culture "permeates and transforms" the human psyche (R. Schweder, in Ratner et al., 2003). Greenfield, Maynard, and Marti (this issue) provide a good example of the ways in which qualitative methods make culture more "concrete". These authors show how activity settings analysis - the detailed observation of what actors do in particular, structured situations-- can explain long-term cultural and social change. Drawing partly on field notes and observations from several decades of field work in a rural Mexican community, the study shows how the nature of specific activities in the economic sphere are linked to shifts in interpersonal and social roles, especially among women, in a traditional community.

Lewis and Ozaki (this issue) provide a second example of the way in which qualitative methods can shed light on the specific ways culture shapes psychological variables in their cross cultural study of emotion terms. A much-researched emotion term in Japanese, amae (emotional dependence) has been a focus in the debate regarding the universality of emotion across cultures. The authors use a clever design to compare the experience of amae among Japanese subjects to the experience of "mardy [ness]", a term for babyish dependence common in the north of England. Their use of the componential model of emotion to generate systematic comparisons suggests that despite considerable conceptual and experiential overlap, amae and mardy are experientially distinct. Differences are linked to the contrasting social consequences of amae and mardy behaviors. While the "collectivist" Japanese are amused and forgiving when someone amaeru[s]; mardy behavior in the "individualistic" UK brings down anger and derision on the subject. The authors find that the social reception of amae and mardy shape individual experiences of these emotions, shedding light on one pathway through which collectivism and individualism may shape psychological processes.

\section{The problem of transferability}

A second problem confronting cross-cultural psychology has to do with the transferability of theoretical constructs. Because most theories have been developed by western, middle class psychologists, it has been proposed that they may be ill suited to the study of other groups. Quite apart from the probable truth-value of this critique, it is difficult to use exclusively quantitative methods to generate meaningful data about the degree to which cross cultural theories actually make sense across cultural contexts. For example, what does "acculturation," per se, mean to immigrant research subjects? Is it a meaningful construct? Is it salient? How does it compare in importance to achieving social and economic success? How does the meaning of acculturation vary across different groups and contexts? In contrast to questionnaire studies, qualitative approaches emphasize an in-depth understanding of the experiences and perspectives of research subjects, often relying on 
these perspectives to shape strategies of inquiry within individual studies. Discovery oriented data from qualitative studies can creatively disrupt pat assumptions and provide a basis for the development of new culturally appropriate theories of psychological phenomena.

\section{The problem of measurement}

A major concern among cross-cultural psychologists using quantitative methods is the translation and establishment of conceptual and metric equivalence of research instruments. Cross-cultural researchers have been criticized as insufficiently concerned about the lack of cross-cultural validity of measures (Greenfield, 1997). However, in recent years considerable concern regarding this problem has been voiced among cross-cultural psychologists (Chastiotis et al., 2009). Standard qualitative methods can help to address the problem of the cross-cultural equivalence of research instruments. Qualitative inquiry is useful at the early stage of conceptual development in survey design, when it can help to establish the constructs, and their properties, salient to participants in the field. Once the instrument has been developed, qualitative methods may be used in conjunction with standard survey approaches to enhance the equivalence of measures or to locate problematic areas where equivalence is lacking. Cognitive interviewing, a qualitative approach that focuses on participants' experiences and interpretation of test items, is often used to systematically investigate the validity of survey instruments and can help to establish group differences when used in comparative designs (Hines, 1993).

\section{Conclusion}

The qualitative and mixed methods studies presented in this special issue suggest only some of the diversity that qualitative and mixed methods research bring to the study of crosscultural psychology. We do not mean the papers to represent the pinnacle of achievement in qualitative and mixed methods research. In our opinion, qualitative research itself stands at a crossroads. As the paradigm wars fade into history, qualitative and mixed methods researchers in the empiricist tradition must work harder to develop systematic, powerful, and transparent methods for analyzing data. We look forward to a number of developments in this area: first, the growth of comparative designs in qualitative and mixed methods research; second, the increased use of multiple data sources; and third, the further development of novel designs which combine qualitative and mixed methods to approach new questions of interest.

We invite the readers of JCCP to view these papers as a starting point. What studies would you design to investigate the questions raised in the special issue? What new questions could you address using the methods that we have presented here? We hope the collection will interest and inspire you, suggesting new directions of inquiry that may stimulate your curiosity and, ultimately, expand the horizons of our discipline.

\section{Reference List}

Adamopoulos, J.; Lonner, WJ. Culture and Psychology at a Crossroad: Historical Perspective and Theoretical Analysis. In: Matsumoto, D., editor. The Handbook of Culture and Psychology. Oxford University Press; Oxford: 2001.

Ashworth, P. The origins of qualitative psychology. 2003.

Barry CA, Britten N, Bradley CP, Stevenson FA. Using reflexivity to optimize teamwork in qualitative research. Qual.Health Research. 1999; 9:26-44.

Chasiotis, A.; Breugelmans, S.; van de Vijver, F. Fundamental questions of cross-cultural psychology. Cambridge University Press; Cambridge: (in press) 
Greenfield PM. You can't take it with you: Why ability assessments don't cross cultures. American Psychologist. 1997; 52:1124.

Hines A. Linking qualitative and quantitative methods in cross-cultural survey research: Techniques from cognitive science. American Journal of Community Psychology. 1993; 21:729-746.

Johnson RB, Onwuegbuzie AJ. Mixed methods research: A research paradigm whose time has come. Educational Researcher. 2009; 33:14-36.

Lincoln, Y.; Guba, E. Naturalistic Inquiry. Sage; Newbury Park: 1985.

Maxcy, S. Pragmatic threads in mixed methods research in the social sciences: The search for multiple models of inquiry and the end of the philosophy of formalism. In: Teddlie, C.; Tashakkori, A., editors. Handbook of mixed methods in social and behavioral research. Sage; Thousand Oaks: 2003.

Maxwell J. Causal explanation, qualitative research, and scientific inquiry in education. Educational Researcher. 2004; 33:3-11.

Maxwell, J.; Loomis, D. Mixed methods design: An alternative approach. In: Teddlie, C.; Tashakkori, A., editors. Handbook of mixed methods in social and behavioral research. Sage; Thousand Oaks: 2003.

Mohr, L. Explaining organizational behavior. Jossey-Bass; San Francisco: 1982.

Office of Behavioral and Social Sciences Research. Qualitative methods in health research: Opportunities and considerations in application and review. Culture and Qualitative Research Interest Group, National Institutes of Health; 1999.

Ratner C, Hui L. Theoretical and methodological problems in cross-cultural psychology. [References]. Journal for the Theory of Social Behaviour. 2003; 33(1):203.

Strauss, A.; Corbin, J. Basics of qualitative research: Techniques and procedures for developing grounded theory. 2nd ed. Sage; Thousand Oaks: 1998.

Teddlie, C.; Tashakkori, A. Mixed methodology: Combining qualitative and quantitative approaches. Sage; Thousand Oaks: 1998.

Tesch, R. Qualitative research: analysis types and software tools. Chapter 1: History of qualitative research. The Falmer Press; Basingstoke: 2009.

Wundt, W. Elements of folk psychology--Outline of a psychological history of the development of mankind. Blakiston Press; San Francisco: 2008. 